\title{
Preparing projected entangled pair states on a quantum computer
}

\author{
Martin Schwarz, Kristan Temme, and Frank Verstraete \\ Vienna Center for Quantum Science and Technology, \\ Faculty of Physics, University of Vienna, Vienna, Austria
}

\begin{abstract}
We present a quantum algorithm to prepare injective PEPS on a quantum computer, a class of open tensor networks representing quantum states. The run-time of our algorithm scales polynomially with the inverse of the minimum condition number of the PEPS projectors and, essentially, with the inverse of the spectral gap of the PEPS' parent Hamiltonian.
\end{abstract}

Projected Entangled Pair States, or PEPS [2], have been proposed as a class of quantum states especially suited to describe the ground states of local Hamiltonians in quantum many-body physics. PEPS are a higherdimensional generalization of the one-dimensional Matrix Product States [3], or MPS, for which many interesting properties have been proven: For example, MPS provably approximate the ground state of 1D local Hamiltonians with constant spectral gap [4, 5], exhibit an area law [5] as well as an exponential decay of two-point correlation functions. Furthermore, for each MPS with the injectivity property [6], a parent Hamiltonian can be constructed with this MPS as its unique ground state. MPS can also be prepared efficiently on a quantum computer [7]. PEPS however form a much richer class of states, and can e.g. represent critical systems and systems with topological quantum order [1]. It is conjectured that all ground states of gapped local Hamiltonians in higher dimensions can be represented faithfully as PEPS, and although there are strong indications for this fact, this has not been proven. What is clear, however, is the fact that one can also construct parent Hamiltonians for them [6], and the PEPS will be the unique ground states of those Hamiltonians if the PEPS obeys the so-called injectivity condition [6]. Many physically relevant classes of PEPS on lattices are known to be almost always injective, including e.g. the 2D AKLT state [6]. A particularly interesting subclass of PEPS is the one that consists of all those states whose parent Hamiltonian have a gap that scales at most as an inverse polynomial as a function of the system size: in that case, a local observable (i.e. the local Hamiltonian) allows to distinguish the state from all other ones, as the ground state always has energy zero by construction. It was an open problem [1] whether such states could however be even created on a quantum computer, as an algorithm that would allow to prepare any PEPS would allow for the solution of $P P$-complete problems [8].

In this article we show how well-conditioned injective PEPS can be prepared on a quantum computer efficiently. The key idea of our approach is to grow the PEPS step by step. We demand that not only our final PEPS is the unique ground state of its parent local Hamiltonian, but also that there exists a sequence of partial sums of the local terms of the parent Hamiltonian, such that each partial sum has a unique ground state of its own. Based on this assumption, the algorithm starts with a physical realization of the valence bond pairs as its initial state and iteratively performs entangling measurements on the virtual particles to map virtual degrees of freedom to physical ones, just as in the definition of the PEPS. The PEPS is called injective, iff this map is (left) invertible which can only be the case if the dimension of the physical space is actually at least as large as the dimension of the virtual space at each vertex. Preparing a PEPS by measurements may seem to require post-selection to project onto the right measurement outcome. To overcome this issue we use the Marriott-Watrous trick [9, 10] of undoing a measurement based on Jordan's lemma [11] and combine it with the uniqueness property of injective PEPS [6] to prepare the required eigenstates. A key element that contributes to the success of this algorithm is the fact that the measurements are not done locally, such as in the framework of dissipative quantum state engineering [12], but globally by running a phase estimation algorithm that singles out the ground subspace; a similar approach was used in the context of the quantum Metropolis sampling algorithm [13]. Alternatively, methods for eigenpath traversal $[17,18]$ can also be applied [21].

Definitions and Results.-Before stating the result, we review the definition of PEPS and their essential properties. Recall $[2,6]$ that PEPS are quantum states defined over an arbitrary graph $G=(V, E)$ such that quantum systems of local dimension $d$ are assigned to each vertex. We construct the PEPS by assigning to each edge $e \in E$ a maximally entangled state $\sum_{i=1}^{D}|i i\rangle$. In this way, a vertex $v \in V$ with degree $k$ gets associated with $k$ virtual $D$-dimensional systems. Finally, a map $A^{(v)}: \mathbb{C}^{D} \otimes \mathbb{C}^{D} \otimes \cdots \mathbb{C}^{D} \mapsto \mathbb{C}^{d}$ is applied to each vertex, taking the $k$ virtual $D$-dimensional systems to a single physical $d$-dimensional system. The linear map $A^{(v)}$ is usually called the PEPS "projector" and is parameterized by tensors $A_{i}^{(v)}$ as follows: $A^{(v)}=\sum_{i=1}^{d} \sum_{j_{1}, \ldots, j_{k}=1}^{D} A_{i, j}^{(v)}|i\rangle\left\langle j_{1}, \ldots, j_{k}\right|$ where $A_{i}^{(v)}$ is a tensor with $k$ indices. The PEPS can now be written as $|\psi\rangle=\sum_{i_{1}, \ldots, i_{n}=1}^{d} \mathcal{C}\left[\left\{A_{i_{v}}^{(v)}\right\}_{v}\right]\left|i_{1}, \ldots, i_{n}\right\rangle$ where $\mathcal{C}$ means the contraction of all tensors $A_{i}^{(v)}$ according to the edges of the graph. In the most general case the virtual index dimension $D$ as well as the physical index dimension $d$ may also depend on the edges $e$ and vertices $v$ of the interaction graph, but we suppress this detail in favor of simplicity. Note, that w.l.o.g. $A^{(v)} \geq 0$ may be assumed, 
since for arbitrary $\tilde{A}^{(v)}$ we can choose a local basis by performing a polar decomposition, i.e. $\tilde{A}^{(v)}=U^{(v)} A^{(v)}$ with $U^{(v)}$ unitary and $A^{(v)} \geq 0$.

A PEPS $|\psi\rangle$ is called injective [6], if each PEPS projector $A^{(v)}$ has a left inverse. For some PEPS this may only be true, after some local contractions of a constant number of PEPS tensors $A^{(v)}$ according to the interaction graph of the PEPS forming new projectors $\hat{A}^{(v)}$ for which the condition above holds. Since this blocking can be performed efficiently for constant degree graphs, we may assume for the remainder of this paper, that it has already been performed, such that each individual $A^{(v)}$ in our input is already injective by itself. Note, that the existence of a left inverse allows us to strengthen the assumption $A^{(v)} \geq 0$ w.l.o.g. to $A^{(v)}>0$ for all $v$.

For injective PEPS, there is a simple construction [6] of a 2-local parent Hamiltonian, such that the injective PEPS is its unique, zero-energy ground state. This construction gives a parent Hamiltonian for a quantum system consisting of $n$ particles with $d$-dimensional Hilbert spaces.

Let $H$ be a Hermitian matrix with $\lambda_{0}<\lambda_{1}$ its smallest and second smallest eigenvalues. Then we call $\Delta(H)=$ $\lambda_{1}-\lambda_{0}$ the spectral gap of $H$. For any matrix $A$, the condition number $\kappa(A)$ is defined as $\kappa(A)=\frac{\sigma_{\max }(A)}{\sigma_{\min }(A)}$, where $\sigma_{\max }(A)$ and $\sigma_{\min }(A)$ are the largest and smallest singular values of $A$, respectively. We are now in a position to state the performance of our algorithm as our main theorem:

Theorem 1. Let $G=(V, E)$ be an interaction graph with bounded degree and some total order defined on $V$. Let $\left\{A^{(v)}\right\}_{v \in V_{[t]}}$ be a set of injective PEPS projectors of dimension $d \times D^{k}$ associated with each $v$ in $V$ up to vertex $t$ (according to the total vertex order) describing a sequence of PEPS $\left|\psi_{t}\right\rangle$, and let $\kappa=\max _{v \in V} \kappa\left(A^{(v)}\right)$ be the largest condition number of all PEPS projectors. Let $\Delta=$ $\min _{t} \Delta\left(H_{t}\right)$, where $\Delta\left(H_{t}\right)$ is the spectral gap of the parent Hamiltonian $H_{t}$ of the PEPS $\left|\psi_{t}\right\rangle$. Then there exists a quantum algorithm generating the final PEPS $\left|\psi_{|V|}\right\rangle$ with probability at least $1-\varepsilon$ in time $\tilde{O}\left(\frac{|V|^{2}|E|^{2} \kappa^{2}}{\varepsilon \Delta}+|V| k d^{6}\right)$.

Algorithm. - Conceptually, PEPS are constructed by first preparing entangled pair states $|\psi\rangle=\sum_{i}|i i\rangle$ for each edge of the interaction graph describing the PEPS, and then projecting the $k$ virtual indices associated with each vertex to a single physical index. While this construction is usually considered only a theoretical device, the proposed algorithm is indeed simulating the above construction for the case of injective PEPS with gapped Hamiltonians. This entails making the virtual indices physical as well.

Figure 1 presents our algorithm in pseudo-code. We proceed by explaining each step in detail. PEPS construction starts in step 2 by distributing maximally entangled states of the desired bond dimension according to the interaction graph $G=(E, V)$. The resulting system is the zero-energy ground state of a simple Hamil-
Input: Interaction graph $G=(V, E)$ with degree bound $k$ and total vertex order. For each $v \in V, d \times D^{k}$-matrices $A^{(v)}$ as PEPS projectors. Acceptable probability of failure $\varepsilon$. Output: PEPS $|\psi\rangle$ with probability at least $1-\varepsilon$.

1. $t \leftarrow 0$

2. $\left|\psi_{t}\right\rangle \leftarrow$ entangled pair for each edge $e \in E$.

3. $H_{t}=\sum_{e \in E} H_{e}$

4. For each $v \in V$ according to total order:

(a) $H_{t+1} \leftarrow H_{t}$

(b) For each neighbor $v^{\prime} \in V$ of $v$ :

- remove term $H_{t}^{\left(v, v^{\prime}\right)}$ from $H_{t+1}$

- compute parent Hamiltonian term $H_{t+1}^{\left(v, v^{\prime}\right)}$ using $A^{(v)}$

- add term $H_{t+1}^{\left(v, v^{\prime}\right)}$ to $H_{t+1}$

(c) Add $H_{p h y}^{(v)}=c\left(\mathbb{1}-P_{p h y}^{(v)}\right)$ to $H_{t+1}$

(d) $\left|\psi_{t+1}^{(\perp)}\right\rangle \leftarrow$ measure $H_{t+1}$ on $\left|\psi_{t}\right\rangle$

(e) While measured energy nonzero:
i. $\left|\psi_{t}^{(\perp)}\right\rangle \leftarrow$ measure $H_{t}$ on $\left|\psi_{t+1}^{(\perp)}\right\rangle$
ii. $\left|\psi_{t+1}^{(\perp)}\right\rangle \leftarrow$ measure $H_{t+1}$ on $\left|\psi_{t}^{(\perp)}\right\rangle$

(f) $t \leftarrow t+1$

FIG. 1. Algorithm constructing injective PEPS

tonian $H_{0}$ consisting purely of terms projecting onto $H_{e}=\mathbb{1}-\frac{1}{d} \sum_{i, j=1}^{d}|i i\rangle\langle j j|$ for each edge of the interaction graph (step 3). Note, that this simple Hamiltonian is gapped.

We now describe the main iteration of the algorithm (step 4), which is illustrated in figure 2. In steps $4 \mathrm{a}-4 \mathrm{c}$, after having selected the next vertex $v$ of the interaction graph according to the total vertex order, we construct a new Hamiltonian $H_{t+1}$ from $H_{t}$ : First, we select a $d$-dimensional "physical" subspace from the $D^{k}$ dimensional space at each vertex $v$. This subspace is represented by projector $P_{p h y}^{(v)}$. Then we remove for each neighboring vertex $v^{\prime}$ of $v$ the term $H_{t}^{\left(v, v^{\prime}\right)}$. These are either trivial $H_{e}$ terms or temporary boundary terms (see below). Next we compute the new parent Hamiltonian terms $H_{t+1}^{\left(v, v^{\prime}\right)}$ according to [6] and add them to $H_{t+1}$ reflecting the application of $A^{(v)}$. Restricted to the "physical" subspace $P_{p h y}^{(v)}$, each $H^{\left(v, v^{\prime}\right)}$ is simply a sum of 2-local terms over all edges $e$ from $v$ to vertices $v^{\prime}$. Note, that parent Hamiltonian terms $H^{\left(v, v^{\prime}\right)}$ towards any open "virtual index" $v^{\prime}$ are only temporary boundary terms which are computed in exactly the same way just as those for any other vertex by assuming the identity as the applicable PEPS map. Since the identity is trivially invertible, each intermediate PEPS is also injective and thus the unique ground state of the intermediate Hamitonian $H_{t+1}$. Since the "physical" $d$-dimensional space is just a subspace of the $D^{k}$ "virtual" space that is in fact also im- 

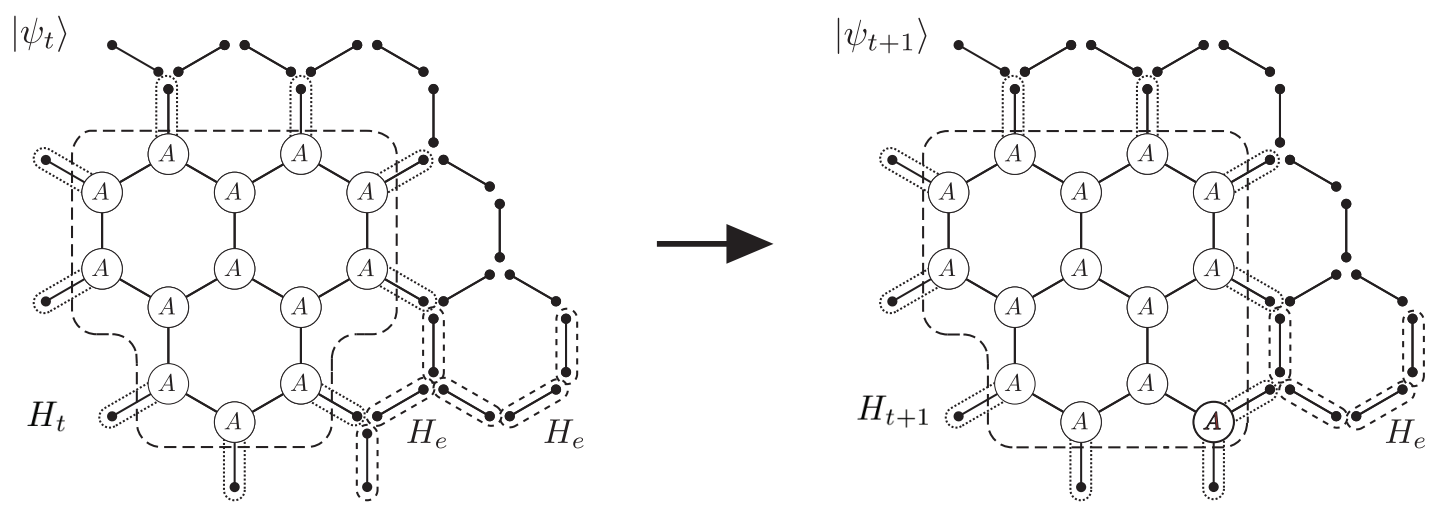

FIG. 2. In each step, the algorithm processes one vertex $v: H_{t}$ is grown into $H_{t+1}$ by removing all existing terms referring to $v$, before the $2 k$-local parent Hamiltonian terms are added implementing PEPS projector $A^{(v)}$ at $v$. Note, that terms around $v$ connecting to an open "virtual" index $v^{\prime}$ (bonds with dotted border) are only temporary and are removed in later steps of the algorithm. All terms constraining a vertex $v$ only restrict the physical subspace $P_{p h y}^{(v)}$, while degrees of freedom in the orthogonal subspace $\left(\mathbb{1}-P_{p h y}^{(v)}\right)$ are eliminated with an additional penalty term $H_{p h y}^{(v)}$ that is added per vertex.

plemented physically in this algorithm, $H^{(v)}$ is actually a sum of $2 k$-local projectors. In order to ensure we produce a state with a single $d$-dimensional local space associated to each vertex $v$ in the final PEPS, we add an extra term $H_{p h y}^{(v)}=c\left(\mathbb{1}-P_{p h y}^{(v)}\right)$ in this step. This term penalizes the orthogonal complement of the chosen subspace with some energy $c \gg \Delta$.

Note, that prior to the execution of step $4 \mathrm{~d}$, the system is in the ground state $\left|\psi_{t}\right\rangle$ of $H_{t}$ by construction. This ground state is unique by the injectivity assumption we make for each intermediate PEPS $\left|\psi_{t}\right\rangle$ prepared in each iteration. In order to transition to the ground state $\left|\psi_{t+1}\right\rangle$ of $H_{t+1}$, we run the phase estimation [14] algorithm for Hamiltonian $H_{t+1}$, perform a binary measurement to project $\left|\psi_{t}\right\rangle$ onto the zero/non-zero energy subspaces of $H_{t+1}$, and uncompute the phase estimation (step $4 \mathrm{~d}$ ). This step requires an inverse eigenvalue gap $\Delta^{-1}$ between these two subspaces that scales with $O($ poly $(|V|))$ for the phase estimation to be efficient and precise enough [15]. We assume that such a gap exists for each intermediate parent Hamiltonian $H_{t}$ that we construct according to the total vertex order defined on the interaction graph.

If the measurement results in the projection onto the zero-energy subspace of $H_{t+1}$ we proceed to the next iteration (step 4e). By Lemma 2, this event occurs with probability at least $\kappa\left(A^{(v)}\right)^{-2}$, where $\kappa\left(A^{(v)}\right)$ is the condition number of PEPS projector $A^{(v)}$ associated with vertex $v$. Note, that the injectivity property of the PEPS assures, that each $\kappa\left(A^{(v)}\right)$ is a positive constant. If the measurement projects onto the excited subspace of $H_{t+1}$, we undo the measurement by measuring $H_{t}$ again (step $4(\mathrm{e}) \mathrm{i})$. If this second measurement results in a projection on the ground state, we have exactly undone the (unsuccessful) measurement of $H_{t+1}$, otherwise the system is in the excited subspace of $H_{t}$. In both cases the projection onto the groundstate of $H_{t+1}$ can now be at- tempted again, with success probabilities $\kappa\left(A^{(v)}\right)^{-2}$ and $1-\kappa\left(A^{(v)}\right)^{-2}$, respectively (step 4(e)ii). By Lemma 3, the inner loop will succeed in projecting onto the ground state of $H_{t+1}$ with probability at least $1-\frac{1}{2 e s}$ after at most $\kappa^{2} s$ attempts, with $s$ chosen as $s=\frac{|V|}{2 e \varepsilon}$. Once all $|V|$ vertices have been covered, the outer loop terminates with the PEPS $|\psi\rangle$ in its output register with probability at least $1-\varepsilon$, as shown in Theorem 1 .

Bounding the transition probabilities. - As a first step in our analysis, we need a lower bound on the transition probability from $\left|\psi_{t}\right\rangle$ to $\left|\psi_{t+1}\right\rangle$. To this end we proof the following lemma.

Lemma 2. Let $\left|\psi_{t}\right\rangle=\frac{1}{\sqrt{Z_{t}}}\left|A_{t}\right\rangle$ be the normalized PEPS $\left|A_{t}\right\rangle$, where $\left|A_{t}\right\rangle$ is the unnormalized partial PEPS resulting from the contraction of PEPS projectors $A^{(v)}$ for all vertices $v$ processed in the algorithm up to step $t$ and let $Z_{t}=\left\langle A_{t} \mid A_{t}\right\rangle$. Let $\left|A_{t+1}\right\rangle=A_{t+1}\left|A_{t}\right\rangle$ where $A_{t+1}$ is the PEPS projector of time step $t+1$. Then $\left|\left\langle\psi_{t+1} \mid \psi_{t}\right\rangle\right|^{2} \geq \frac{1}{\kappa\left(A_{t+1}\right)^{2}}>0$.

Proof. A simple calculation shows

$$
\begin{aligned}
\left\langle\psi_{t+1} \mid \psi_{t}\right\rangle & =\frac{1}{\sqrt{Z_{t}}} \frac{1}{\sqrt{Z_{t+1}}}\left\langle A_{t}\left|A_{t+1}^{\dagger}\right| A_{t}\right\rangle \\
& \geq \frac{1}{\sqrt{Z_{t}}} \frac{1}{\sqrt{Z_{t+1}}} \frac{\left\langle A_{t}\left|A_{t+1}^{\dagger} A_{t+1}\right| A_{t}\right\rangle}{\sigma_{\max }\left(A_{t+1}\right)} \\
& =\frac{1}{\sqrt{Z_{t}}} \frac{1}{\sqrt{Z_{t+1}}} \frac{Z_{t+1}}{\sigma_{\max }\left(A_{t+1}\right)} \\
& =\frac{1}{\sigma_{\max }\left(A_{t+1}\right)}\left(\frac{Z_{t+1}}{Z_{t}}\right)^{\frac{1}{2}}
\end{aligned}
$$

where the inequality follows from the operator inequalities $A_{t+1} \geq 0$ and $\frac{A_{t+1}}{\sigma_{\max \left(A_{t+1}\right)}} \leq \mathbb{1}$. This implies

$$
\left|\left\langle\psi_{t+1} \mid \psi_{t}\right\rangle\right|^{2} \geq \frac{1}{\sigma_{\max }\left(A_{t+1}\right)^{2}} \frac{Z_{t+1}}{Z_{t}}
$$


But

$$
\begin{aligned}
Z_{t+1}=\left\langle A_{t}\left|A_{t+1}^{2}\right| A_{t}\right\rangle & \geq \sigma_{\min }\left(A_{t+1}\right)^{2}\left\langle A_{t} \mid A_{t}\right\rangle \\
& =\sigma_{\min }\left(A_{t+1}\right)^{2} Z_{t} .
\end{aligned}
$$

Thus Eq. 5 and Eq. 7 yield the claim

$$
p=\left|\left\langle\psi_{t+1} \mid \psi_{t}\right\rangle\right|^{2} \geq\left(\frac{\sigma_{\min }\left(A_{t+1}\right)}{\sigma_{\max }\left(A_{t+1}\right)}\right)^{2}=\frac{1}{\kappa\left(A_{t+1}\right)^{2}}
$$

Finally, the injectivity assumption of PEPS $\left|\psi_{t+1}\right\rangle$ implies left invertibility of $A_{t+1}$ for each $v$, thus $\kappa\left(A_{t+1}\right)$ is finite, therefore $p>0$.

Bounding the convergence rate. - In this section we analyze the termination probability of the loop at step $4 \mathrm{e}$.

Lemma 3. Let $H_{t}, H_{t+1}$ be Hamiltonians with unique zero-energy groundstates $\left|\psi_{t}\right\rangle$ and $\left|\psi_{t+1}\right\rangle$, respectively. Let $s$ be a positive integer. If the system is in state $\left|\psi_{t}\right\rangle$ initially, then the measurement process alternatingly measuring $H_{t+1}$ and $H_{t}$ and stopping once $\left|\psi_{t+1}\right\rangle$ is reached, takes the system to state $\left|\psi_{t+1}\right\rangle$ with probability at least $1-\frac{1}{2 e s}$ after at most $s / p$ alternations, where $p=\left|\left\langle\psi_{t+1} \mid \psi_{t}\right\rangle\right|^{2}$.

Proof. Let $P, Q$ be the ground state projectors of $H_{t}$ and $H_{t+1}$, respectively, and let $P^{\perp}=\mathbb{1}-P, Q^{\perp}=\mathbb{1}-Q$. By Jordan's Lemma, there exists an orthonormal basis in which the Hilbert space decomposes into (1) twodimensional subspaces $S_{i}$ invariant under both, $P$ and $Q$, and (2) one-dimensional subspaces $T_{j}$ on which $P Q$ is either an identity- or zero-projector [10].

Since we know that $\left|\psi_{t}\right\rangle$ and $\left|\psi_{t+1}\right\rangle$ are the unique 1eigenstates of $P$ and $Q$ with overlap $\sqrt{p}$, exactly one $S_{i}$ is relevant to our analysis. This two-dimensional subspace is spanned by both, $\left|\psi_{t}\right\rangle$ and some $\left|\psi_{t}^{\perp}\right\rangle$, as well as by $\left|\psi_{t+1}\right\rangle$ and some $\left|\psi_{t+1}^{\perp}\right\rangle$. Among these four vectors, we have got the following relationships [9]:

$$
\begin{aligned}
\left|\psi_{t}\right\rangle & =-\sqrt{p}\left|\psi_{t+1}\right\rangle+\sqrt{1-p}\left|\psi_{t+1}^{\perp}\right\rangle \\
\left|\psi_{t}^{\perp}\right\rangle & =\sqrt{1-p}\left|\psi_{t+1}\right\rangle+\sqrt{p}\left|\psi_{t+1}^{\perp}\right\rangle \\
\left|\psi_{t+1}\right\rangle & =-\sqrt{p}\left|\psi_{t}\right\rangle+\sqrt{1-p}\left|\psi_{t}^{\perp}\right\rangle \\
\left|\psi_{t+1}^{\perp}\right\rangle & =\sqrt{1-p}\left|\psi_{t}\right\rangle+\sqrt{p}\left|\psi_{t}^{\perp}\right\rangle
\end{aligned}
$$

Considering these symmetrical relations, we see that alternating measurements of $H_{t}$ and $H_{t+1}$ generate a Markov process among these four states. Since the process terminates whenever it hits $\left|\psi_{t+1}\right\rangle$, the only histories which can keep the process from terminating are those with an initial transition $\left|\psi_{t}\right\rangle \rightarrow\left|\psi_{t+1}^{\perp}\right\rangle$ and which then keep repeating either one of the following two pairs of transitions

$$
\begin{aligned}
\left|\psi_{t+1}^{\perp}\right\rangle \rightarrow\left|\psi_{t}\right\rangle & \rightarrow\left|\psi_{t+1}^{\perp}\right\rangle \\
\left|\psi_{t+1}^{\perp}\right\rangle \rightarrow\left|\psi_{t}^{\perp}\right\rangle & \rightarrow\left|\psi_{t+1}^{\perp}\right\rangle,
\end{aligned}
$$

which occur with probabilities $(1-p)^{2}$ and $p^{2}$, respectively. Thus the process terminates after at most $2 m+1$ measurements with probability

$$
p_{\text {term }}(p, m)=1-(1-p)\left(p^{2}+(1-p)^{2}\right)^{m} .
$$

To lower-bound this probability we upperbound $\quad p_{\text {fail }}(p, m)=1-p_{\text {term }}(p, m)$ as $p_{\text {fail }}(p, m) \leq(1-p) \exp (-2 m p(1-p))$ which follows from $(1-q)^{m} \leq e^{-q m}$, for $0 \leq q \leq 1$ and $m \geq 0$. Finally we choose $m$ as a multiple of $\frac{1}{p}$ and find $p_{\text {fail }}(p, s / p) \leq \frac{1}{2 e s}$, which can be seen by straightforward calculus.

Proof of Theorem 1. We complete the proof of Theorem 1 by using Lemma 3 for bounding the failure probability $p_{\text {fail }}$ of the inner loop to derive a lower bound on the success probability of the outer loop over all vertices in $V$. That is, we have to show that $\left(1-p_{\text {fail }}\right)^{|V|} \geq 1-\varepsilon$. Since $\left(1-p_{\text {fail }}\right)^{|V|} \geq 1-|V| p_{\text {fail }}$ by truncating higher-order terms from the binomial series and assuming $|V|>1$ it suffices to show $|V| p_{\text {fail }} \leq \varepsilon$. Using Lemma 3, we find the first inequality of $|V| p_{\text {fail }} \leq \frac{|V|}{2 e s} \leq \varepsilon$, while the second inequality is satisfied by choosing $s \geq \frac{|V|}{2 e \varepsilon}$. Thus, for the algorithm to succeed with at least probability $1-\varepsilon$ we have to choose $m \geq \frac{s}{p} \geq \frac{|V|}{2 p e \varepsilon}$. Since we know from Lemma 2 that $p \geq \frac{1}{\kappa^{2}}$, choosing $m \geq \frac{\kappa^{2}|V|}{2 e \varepsilon} \geq \frac{|V|}{2 p e \varepsilon}$ suffices. Thus the inner loop performs at most $2 m+1 \leq \frac{\kappa^{2}|V|}{e \varepsilon}$ measurements. The outer loop iterates over $|\bar{V}|$ vertices, thus the total number of measurements is less than $\frac{\kappa^{2}|V|^{2}}{e \varepsilon}+|V|$. Bookkeeping of the active Hamiltonian terms in the outer loop requires a total time of $O(|V| k)$ using simple arrays as data structures, and $O\left(|V| k d^{6}\right)$ to compute all parent Hamiltonian terms, both of which are dominated by the $O\left(|V|^{2}\right)$ time of the inner loop for small $d$. Finally, since each phase estimation step requires $\tilde{O}\left(|E|^{2} / \Delta\right)[10,14,15]$, where $\tilde{O}(\cdot)$ suppresses more slowly growing factors such as $\exp (\sqrt{\ln (|E| / \Delta)})$ [16], we find a total runtime of $\tilde{O}\left(\frac{|V|^{2}|E|^{2} \kappa^{2}}{\varepsilon \Delta}+|V| k d^{6}\right)$. This completes the proof of Theorem 1 .

Conclusion. - In this Letter we have shown how to construct quantum states described by injective PEPS in polynomial time by first reducing the problem to the generation of a sequence of unique ground states of certain Hamiltonians and then preparing that sequence. In future work we will focus on extending the class of preparable PEPS and possible performance improvements following from the results of [18-20].

This work has been supported by Austrian SFB project FoQuS F4014. 
[1] F. Verstraete, M. Wolf, D. Perez-Garcia, and J. Cirac, Physical review letters 96, 220601 (2006).

[2] F. Verstraete and J.I. Cirac, Physical Review A 70, 060302(R) (2004), arXiv:quant-ph/0311130.

[3] S. Rommer and S. Östlund, Physical Review B 55, 2164 (1997).

[4] F. Verstraete and J.I. Cirac, Physical Review B 73, 094423 (2006).

[5] M. Hastings, Journal of Statistical Mechanics: Theory and Experiment 2007, P08024 (2007).

[6] D. Perez-Garcia, F. Verstraete, J. I. Cirac, and M. M. Wolf, Quant. Inf. Comp 8, 0650 (2008), arXiv:0707.2260.

[7] C. Schön, E. Solano, F. Verstraete, J.I. Cirac, and M. Wolf, Physical review letters 95, 110503 (2005).

[8] N. Schuch, M. Wolf, F. Verstraete, and J.I. Cirac, Physical review letters 98, 140506 (2007).

[9] C. Marriott and J. Watrous, Computational Complexity 14, 122 (2005).

[10] D. Nagaj, P. Wocjan, and Y. Zhang, QIC 9, 1053 (2009), arXiv:0904.1549.

[11] C. Jordan, Bull. Soc. Math. France 3, 103 (1875).
[12] F. Verstraete, M. Wolf, and J. Cirac, Nature Physics 5, 633 (2009), arXiv:0803.1447.

[13] K. Temme, T. Osborne, K. Vollbrecht, D. Poulin, and F. Verstraete, Nature 471, 87 (2011).

[14] M. Nielsen and I. Chuang, Quantum Computation and Quantum Information (Cambridge University Press, Cambridge, 2000).

[15] D. Berry, G. Ahokas, R. Cleve, and B. Sanders, Communications in Mathematical Physics 270, 359 (2007), 10.1007/s00220-006-0150-x.

[16] A. Harrow, A. Hassidim, and S. Lloyd, Physical review letters 103, 150502 (2009).

[17] D. Aharonov and A. Ta-Shma, SIAM Journal on Computing 37, 47 (2007).

[18] S. Boixo, E. Knill, and R. Somma, Arxiv preprint arXiv:1005.3034 (2010)

[19] S. Boixo and R.D. Somma, Physical Review A 81, 032308 (2010).

[20] R. Somma and S. Boixo, Arxiv preprint arXiv:1110.2494 (2011).

[21] S. Boixo, personal communication (2011). 